\title{
ASCA Observations of the Composite Warm Absorber in NGC 3516
}

\author{
G. A. Kriss ${ }^{1}$, J. H. Krolik ${ }^{1}$, C. Otani ${ }^{2}$, B. R. Espey ${ }^{1}$, T. J. Turner ${ }^{3,4}$, T. Kiii ${ }^{5}$, Z. Tsvetanov ${ }^{1}$, T.

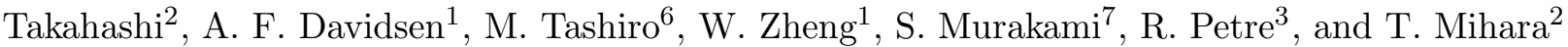

\begin{abstract}
We obtained X-ray spectra of the Seyfert 1 galaxy NGC 3516 in March 1995 using the Japanese X-ray satellite ASCA. Simultaneous far-UV observations were obtained with the Hopkins Ultraviolet Telescope on the Astro-2 shuttle mission. The ASCA spectrum shows a lightly absorbed power law of energy index 0.78. The low energy absorbing column is significantly less than previously seen. Prominent O VII and O VIII absorption edges are visible, but, consistent with the much lower total absorbing column, no Fe K absorption edge is detectable. A weak, narrow Fe K $\alpha$ emission line from cold material is present as well as a broad Fe $\mathrm{K} \alpha$ line. These features are similar to those reported in other Seyfert 1 galaxies. A single warm absorber model provides only an imperfect description of the low energy absorption. In addition to a highly ionized absorber with ionization parameter $U=1.66$ and a total column density of $1.4 \times 10^{22} \mathrm{~cm}^{-2}$, adding a lower ionization absorber with $U=0.32$ and a total column of $6.9 \times 10^{21} \mathrm{~cm}^{-2}$ significantly improves the fit. The contribution of resonant line scattering to our warm absorber models limits the Doppler parameter to $<160 \mathrm{~km} \mathrm{~s}^{-1}$ at $90 \%$ confidence. Turbulence at the sound speed of the photoionized gas provides the best fit. None of the warm absorber models fit to the X-ray spectrum can match the observed equivalent widths of all the UV absorption lines. Accounting for the X-ray and UV absorption simultaneously requires an absorbing region with a broad range of ionization parameters and column densities.
\end{abstract}

Subject headings: galaxies: active — galaxies: individual (NGC 3516) — galaxies: nuclei — galaxies: Seyfert — X-ray: galaxies

\footnotetext{
${ }^{1}$ Department of Physics \& Astronomy, Johns Hopkins University, Baltimore, MD 21218.

${ }^{2}$ The Institute of Physical and Chemical Research (RIKEN), Hirosawa, Wako, Saitama 351-01, Japan.

${ }^{3}$ Laboratory for High Energy Astrophysics, NASA/Goddard Space Flight Center, Greenbelt, MD 20771.

${ }^{4}$ Universities Space Research Association.

${ }^{5}$ Institute of Space and Astronautical Sciences, Yoshinodai, Sagamihara, Kanagawa 229, Japan.

${ }^{6}$ Department of Physics, University of Tokyo, 7-3-1 Hongo, Bunkyo-ku, Tokyo 113, Japan.

${ }^{7}$ Department of Physics, Osaka City University, 3-3-138, Sugimoto, Sumiyoshi-ku, Osaka, 558, Japan.
} 


\section{Introduction}

Intrinsic absorption is a valuable tool for probing structures in active galactic nuclei (AGN). While absorption may in principle arise anywhere in the host galaxy, the most interesting absorbers are those that appear to be associated with the central engine. These "warm absorbers" commonly appear in the X-ray spectra of AGN (Turner et al. 1993; Nandra \& Pounds 1994), and they could be material in the broad-emission-line region (BELR) (e.g. Netzer 1993; Reynolds \& Fabian 1995) or the X-ray heated wind which forms the reflecting region in type 2 AGN (Krolik \& Kriss 1995), or an entirely new component. If X-ray warm absorbers are related to associated UV absorption systems (Mathur et al. 1994; Mathur, Wilkes, \& Elvis 1995), then UV and X-ray observations together place powerful constraints on the ionization structure of the absorber. In the X-ray one can measure the column densities of highly ionized species (e.g. O VII and O VIII) while simultaneously observing lower ionization relatives in the UV (O VI, $\mathrm{N}$ v, and $\mathrm{C}$ IV). Objects with strong UV absorption lines and soft X-ray absorption are therefore good candidates for further tests of this hypothesis.

The Seyfert 1 galaxy NGC 3516 exhibits unusually strong, variable UV absorption lines (Ulrich \& Boisson 1983; Voit, Shull, \& Begelman 1987; Walter et al. 1990; Kolman et al. 1993; Koratkar et al. 1996), and has a variable X-ray spectrum characteristic of the warm absorber phenomenon (Halpern 1982). Observations obtained with Ginga (Kolman et al. 1993; Nandra \& Pounds 1994) show a flat power law with energy index $\sim 0.5$ over the $2-18 \mathrm{keV}$ range, a highly ionized iron edge

with a corresponding total column density of $\sim 3 \times 10^{23} \mathrm{~cm}^{-2}$, and a cold fluorescent $\mathrm{Fe} \mathrm{K} \alpha$ line with $\mathrm{EW}=377 \mathrm{eV}$.

To measure simultaneously the X-ray and UV absorption in NGC 3516 we coordinated ASCA observations with the flight of the Astro-2 space shuttle mission in March 1995. Far-ultraviolet spectra obtained with the Hopkins Ultraviolet Telescope (HUT) that allow us to measure the resonance doublets of O VI, N v, Si IV and C IV are discussed in a companion paper by Kriss et al. (1996).

\section{The ASCA Observations}

Four X-ray telescopes (Serlemitsos et al. 1995) consisting of nested conical foil mirrors image $\mathrm{X}$-rays onto four separate detectors in the ASCA focal plane. Two solid-state imaging spectrometers (SIS0 and SIS1), each consisting of four CCD chips, are sensitive from $\sim 0.4$ to $10.0 \mathrm{keV}$ and have an energy resolution of $\sim 2 \%$ at $6 \mathrm{keV}$ (Burke et al. 1994). Two gas imaging spectrometers (GIS) cover the energy range $0.8-10.0 \mathrm{keV}$ with a resolution of $\sim 8 \%$ at $6 \mathrm{keV}$ (Makishima et al. 1996). The observatory and its performance are described by Tanaka, Inoue, \& Holt (1994).

We observed NGC 3516 twice, each time for roughly half a day with intervals lost due to source occultation by the earth and passage through the South Atlantic Anomaly (SAA). The first observation was on 1995 March 11 from 02:24:39-14:40:48 UT. The second was from 20:03:18 UT 
on 1995 March 12 to 08:11:00 UT on 1995 March 13. The HUT spectra described by Kriss et al. (1996) were obtained near the mid-point of each of these intervals.

The SIS detectors were operated in FAINT 1-CCD mode, but the spectra extracted in FAINT mode showed regions of large pixel-to-pixel variations. Converting the data to BRIGHT mode eliminated these variations, and we used these spectra for the analysis presented here. Experimentation with different data screening criteria showed that bright earth avoidance angles of $>25^{\circ}$ were necessary to achieve consistent results. Default screening criteria were used for the dark earth limb angle $\left(>10^{\circ}\right)$ and for the geomagnetic cutoff rigidity $\left(>6 \mathrm{GeV} \mathrm{c}^{-1}\right)$. A few high count rate intervals adjoining SAA passages were rejected by eliminating regions with count rates exceeding

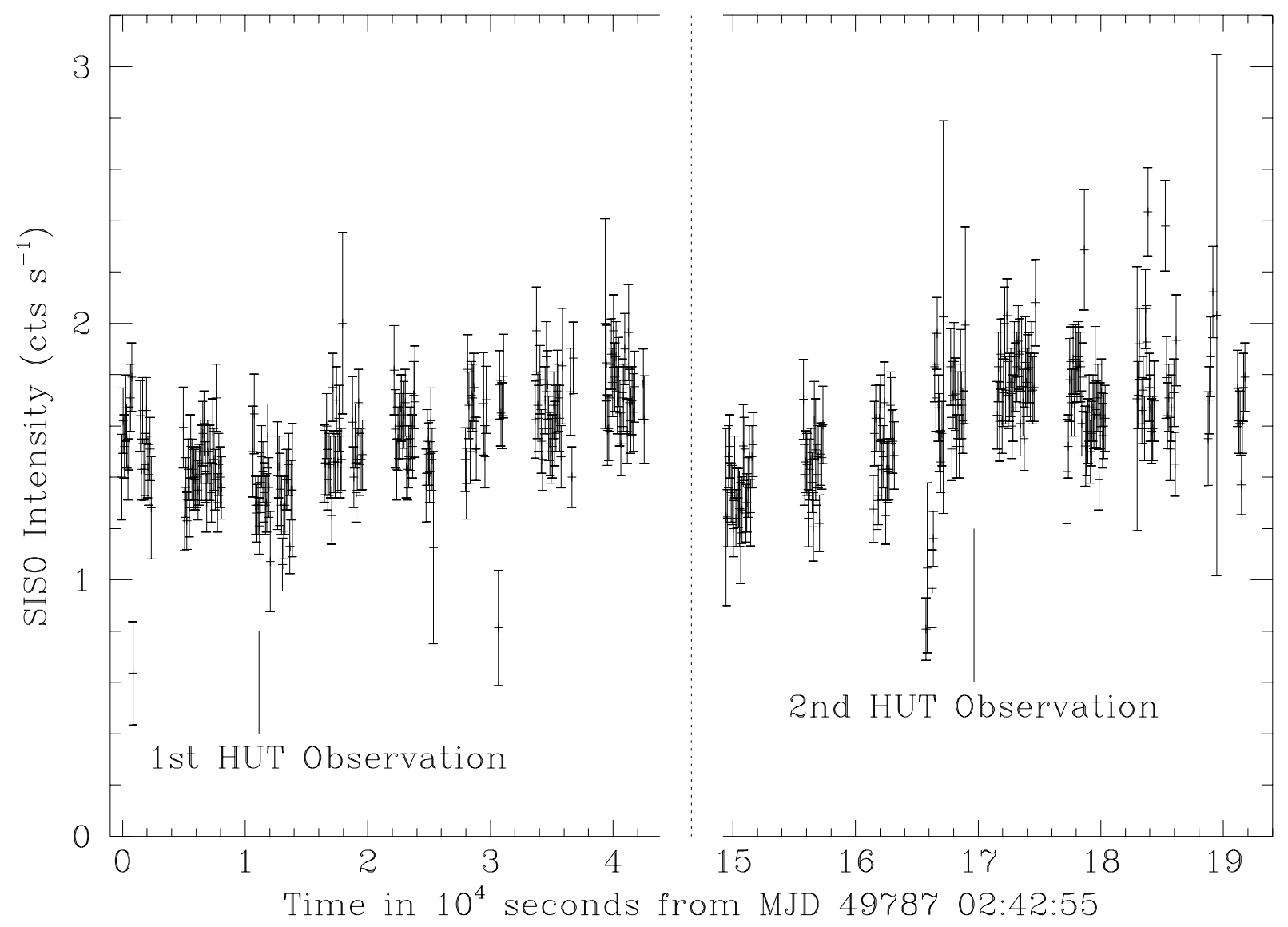

Fig. 1. - The integrated $0.4-10.0 \mathrm{keV}$ count rate of NGC 3516 obtained with ASCA is shown. Time is in seconds from 02:42:55 UT on 11 March 1995. Note the break in the X-axis between the first and second observations. Data are binned into intervals of $100 \mathrm{~s}$. Variations of $\sim 20 \%$ are apparent on timescales of $\sim 20,000 \mathrm{~s}$. The centroids of the integration intervals for the two HUT spectra reported by Kriss et al. (1996) are indicated. 
$2.5 \mathrm{cts} \mathrm{s}^{-1}$. Counts for light curves and spectra were extracted from circular regions centered on NGC 3516 using the largest radius that did not extend beyond the boundary of the CCD (3.5 for SIS0 and 2'.6 for SIS1). As shown by the SIS0 count rate in Figure 1, NGC 3516 showed intensity changes of $\sim 20 \%$ over the course of both observations. The combined data sets yield 54,539 events over a live integration time of 33,534 s in SIS0 and 38,935 events over 33,956 s in SIS1.

\section{Modeling the X-ray Spectrum}

We used the spectral fitting program XSPEC (Shafer, Haberl, \& Arnaud 1989) to model the extracted spectra. To permit the use of $\chi^{2}$ statistics we grouped the data to have a minimum of 25 counts per spectral bin. This affected only bins above $6.5 \mathrm{keV}$. To avoid the worst uncertainties in the detector response, we restricted our fits to bins with energies $0.60 \mathrm{keV}<\mathrm{E}<9.00 \mathrm{keV}$. The SIS0 and SIS1 data were fit jointly.

Although spectral features are obvious in the raw spectrum, we first fit a simple power law with cold absorption to the data to draw a comparison with earlier X-ray observations. This model yields an energy index of 0.62 , a flux at $1 \mathrm{keV}$ of 0.01 photons $\mathrm{cm}^{-2} \mathrm{~s}^{-1} \mathrm{keV}^{-1}$, an equivalent neutral hydrogen column of $N_{H}=1.2 \times 10^{21} \mathrm{~cm}^{-2}$ and $\chi^{2} / \nu=1158 / 435$. The spectral shape and intensity is significantly different from that found in earlier Ginga observations (Kolman et al. 1993). The intensity as observed with ASCA is about twice as high, the spectral index is steeper (0.62 \pm 0.03 compared to a mean of $0.47 \pm 0.04$ in the Ginga data), and the absorbing column $\sim 30 \times$ lower than the value of $\sim 4.06 \pm 0.28 \times 10^{22} \mathrm{~cm}^{-2}$ found by Kolman et al. (1993). As Ginga is sensitive to column densities of $\sim 1 \times 10^{21} \mathrm{~cm}^{-2}$, there is little doubt that the low energy absorption has changed significantly in character since 1989. Such striking differences in column density were seen previously in the Einstein observations of NGC 3516 (Halpern 1982), and they are typical of variations seen in other sources with intrinsic absorption such as NGC 4151 (Yaqoob, Warwick, \& Pounds 1989; Yaqoob et al. 1993) and MR2251-178 (Halpern 1984; Otani et al. (1996) $)$. The spectral index, however, is sensitive to the modeling of the absorption and the iron emission in the spectrum. Using the same Ginga data, Nandra \& Pounds (1994) find a spectral index of 0.65-0.74 and absorption columns of $5.0-7.2 \times 10^{22} \mathrm{~cm}^{-2}$.

The ratio of the SIS0 data to the simple power-law model shown in Figure 2 immediately reveals absorption features below $1 \mathrm{keV}$ due to highly ionized oxygen and strong fluorescent emission from neutral iron around $6.3 \mathrm{keV}$. To characterize these spectral features empirically, we added a succession of Gaussian-profile emission lines and absorption edges (with opacity proportional to $E^{-3}$ ) until we obtained an acceptable fit. An acceptable description of the spectrum requires two low energy absorption edges that we attribute to $\mathrm{O}$ VII and $\mathrm{O}$ vIII, a narrow unresolved Fe $\mathrm{K} \alpha$ emission line, and a broad base to the $\mathrm{Fe} \mathrm{K} \alpha$ line. Table 1 gives the best fit values and $90 \%$ confidence error bars for the parameters of this empirical model.

The broad and narrow Fe K $\alpha$ features have a combined equivalent width (EW) of $253 \pm 109$ 


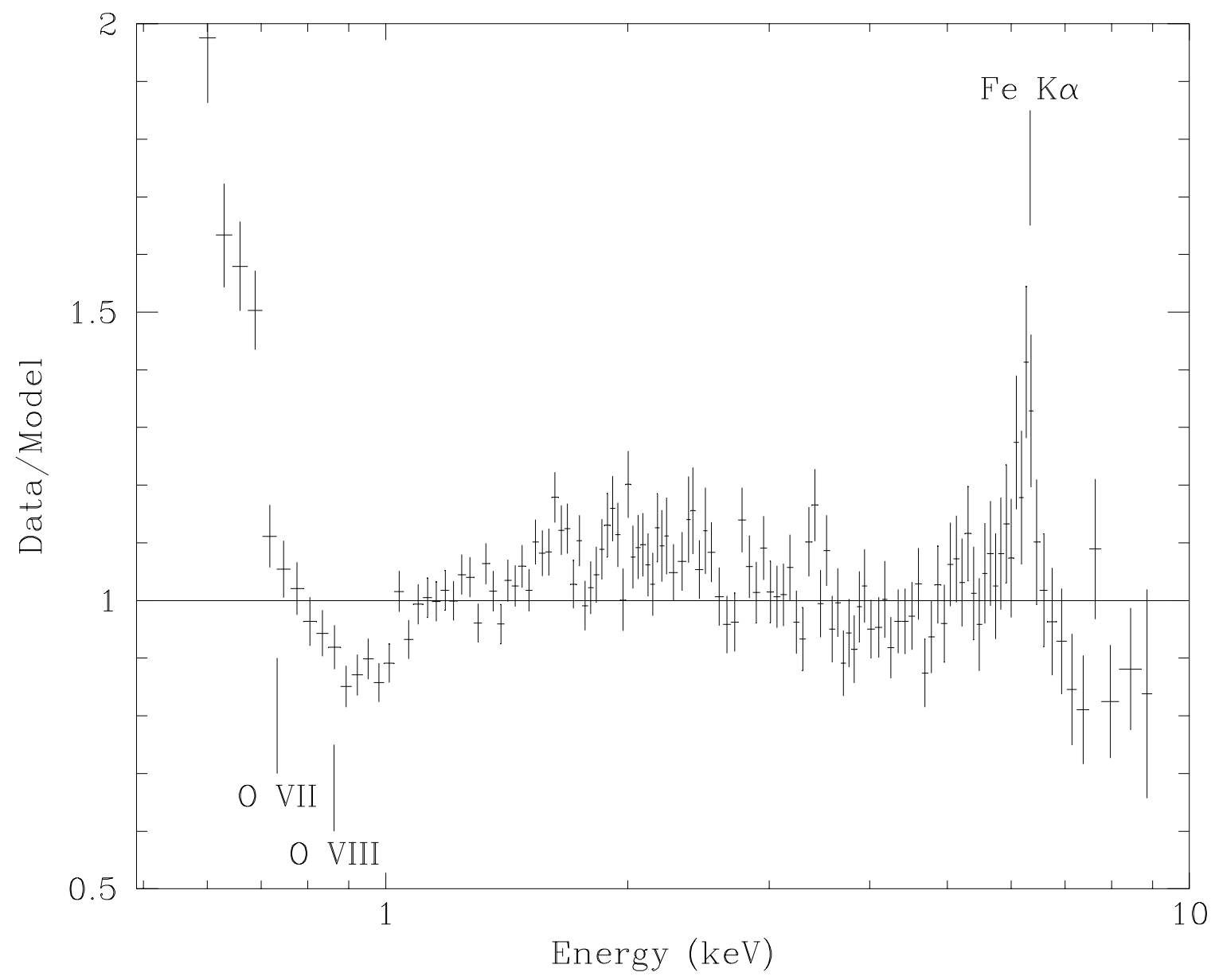

Fig. 2.- To illustrate the features present in the X-ray spectrum of NGC 3516, the data were divided by a simple model consisting of a power law with low energy absorption by neutral gas. The ratio of the data to the model shows a prominent absorption dip around the photoionization edges of $\mathrm{O}$ VII and $\mathrm{O}$ VIII as well as complex structure around the iron $\mathrm{K} \alpha$ line.

$\mathrm{eV}$, comparable to the $377 \pm 44 \mathrm{eV}$ Fe $\mathrm{K} \alpha$ feature in the Ginga spectrum of Kolman et al. (1993). The energies of the oxygen edges and the narrow $\mathrm{Fe} \mathrm{K} \alpha$ line are redshifted relative to the systemic redshift of NGC 3516 ( $z=0.008834$, Vrtilek \& Carleton 1985). The O viI, O viII, and Fe K $\alpha$ features have redshifts of $z=0.046 \pm 0.011, z=0.019 \pm 0.018$, and $z=0.016 \pm 0.0064$, respectively. Inclusion of the two oxygen edges in the fit broadens the distribution of opacity at low energies. This more accurate modeling of the shape of the low energy absorption leads to a spectral index of 0.78 that is steeper than that in the simple power-law fit and is more comparable to the mean power law index of 0.73 found for Ginga observations of Seyferts (Nandra \& Pounds 1994). The residual absorption by cold gas of $N_{H I}=6.8 \times 10^{20} \mathrm{~cm}^{-2}$, however, is still higher than the expected Galactic column of $N_{H}=3.35 \times 10^{20} \mathrm{~cm}^{-2}$ Stark et al. 1992). At first glance it might seem natural to attribute this excess above the Galactic column to cold gas intrinsic to NGC 3516. However, the 
far-UV spectrum obtained with HUT limits the intrinsic neutral hydrogen column in NGC 3516 to $<8 \times 10^{17} \mathrm{~cm}^{-2}$ (Kriss et al. 1996) based on the strengths of the observed Lyman lines and Lyman limit. The most likely explanation for the observed excess cold column is the uncertainty in the ASCA calibration below $1 \mathrm{keV}$. These uncertainties can lead to excess column densities of up to $3 \times 10^{20} \mathrm{~cm}^{-2}$.

Iron edges are also important diagnostics of the ionization state of the absorbing medium. No iron edge feature is apparent in the residuals from our fit, and this is not surprising given the order-of-magnitude lower columns we are seeing relative to earlier X-ray observations of NGC 3516. Adding an additional sharp edge with its energy constrained to be greater than $7.1 \mathrm{keV}$ gives no improvement in $\chi^{2}$. The optical depth at the edge must be less than 0.3 at the $90 \%$ confidence level.

Table 1. Empirical Model Parameter Values

\begin{tabular}{lc}
\hline \multicolumn{1}{c}{ Parameter } & Value $^{\mathrm{a}}$ \\
& \\
\hline$N_{H I}\left(10^{20} \mathrm{~cm}^{-2}\right)^{\mathrm{b}}$ & $6.8 \pm 1.0$ \\
Edge Energy $E_{1}(\mathrm{keV})$ & $0.707 \pm 0.008$ \\
Optical Depth $\tau_{1}$ & $0.65 \pm 0.07$ \\
Edge Energy $E_{2}(\mathrm{keV})$ & $0.855 \pm 0.015$ \\
Optical Depth $\tau_{2}$ & $0.31 \pm 0.05$ \\
Energy index $\alpha$ & $0.776 \pm 0.029$ \\
Normalization $\left(\mathrm{phot} \mathrm{cm}^{-2} \mathrm{~s}^{-1} \mathrm{keV}^{-1}\right)$ & $0.0126 \pm 0.00037$ \\
Narrow Fe Energy $(\mathrm{keV})^{\mathrm{c}}$ & $6.293 \pm 0.040$ \\
Narrow Fe EW $(\mathrm{eV})$ & $73 \pm 27$ \\
Broad Fe Energy $(\mathrm{keV})$ & $5.88 \pm 0.37$ \\
Broad Fe EW $(\mathrm{eV})$ & $180 \pm 106$ \\
Broad Fe FWHM $(\mathrm{keV})$ & $1.58 \pm 0.84$ \\
$\chi^{2} / \nu$ & $494.7 / 426^{\mathrm{d}}$ \\
\hline
\end{tabular}

a The quoted errors are $90 \%$ confidence for a single interesting parameter.

b Equivalent neutral hydrogen column for absorption by cold (neutral) gas.

c The intrinsic width of this unresolved line was held fixed at $0 \mathrm{eV}$. The $90 \%$ confidence upper limit on the line FWHM is $39 \mathrm{eV}$.

$\mathrm{d}$ The probability of exceeding $\chi^{2}$ in this fit is 0.012 . 
The moderately strong edges of highly ionized oxygen in our spectrum naturally suggest an origin in photoionized gas. The dominant strength of the $\mathrm{O}$ VII edge indicates gas of much lower ionization and temperature than that modeled by Krolik \& Kriss (1995), and the absence of an Fe K edge indicates a much smaller column. Accordingly we have computed new models that span the potential range of ionization parameters and column densities with some slight modifications to the procedure described by Krolik \& Kriss (1995). First, we used an ionizing spectrum appropriate

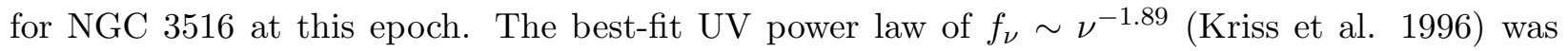
extrapolated to higher frequencies, and the best-fit X-ray power law found here $f_{\nu} \sim \nu^{-0.78}$ was extrapolated to lower frequencies; the two meet at $51 \mathrm{eV}$. Second, the lower temperatures and ionization state place the gas in a regime where thermal equilibrium is more likely because the cooling time is rather shorter. We therefore compute our models in thermal equilibrium. Finally, for ease of comparison to warm absorber models fit to the X-ray spectra of other AGN, we assume constant density clouds and use the ionization parameter $U=n_{\text {ion }} / n_{H}$, where $n_{\text {ion }}$ is the number density of ionizing photons between $13.6 \mathrm{eV}$ and $13.6 \mathrm{keV}$ illuminating the cloud and $n_{H}$ is the density of hydrogen atoms. The transmission of each model is computed exactly as described by Krolik \& Kriss (1995), taking into account resonant line scattering and electron scattering as well as continuum opacity. The resulting models are a two parameter family in total column density $N$ and ionization parameter. These are read into XSPEC as a FITS table for fitting to the X-ray spectrum.

As in Krolik \& Kriss (1995) we assume low density gas $\left(n_{H}=10^{3} \mathrm{~cm}^{-3}\right)$, but there are no density-dependent effects in our calculations or results, provided one considers densities lower than $\sim 10^{11} \mathrm{~cm}^{-3}$. Although the UV continuum in our photoionizing spectrum is steep, we note that there is no lack of high energy photons. The spectrum flattens just below the He II edge to $f_{\nu} \sim \nu^{-0.78}$, and the overall $\alpha_{o x}=1.20$, a value typical of Seyfert 1 galaxies (Kriss \& Canizares 1985). In fact, tests show that our results are rather insensitive to the exact shape of the ionizing spectrum apart from changes in the deduced ionization parameter. (This is a general property of photoionization models with broad distributions of ionizing flux noticed in even the earliest calculations [Tarter, Tucker, \& Salpeter 1969].) For comparison we computed alternative models assuming either an extremely hard spectrum with $f_{\nu} \sim \nu^{-0.78}$ from $2500 \AA$ through the UV and X-ray up to $100 \mathrm{keV}$, or the spectral shape of NGC 5548 as used by Mathur et al. (1995), which contains a soft X-ray excess. Neither of these match the observed broad-band spectral shape, yet they both provide equally good descriptions of the opacity of the warm absorber.

The $\mathrm{Fe} \mathrm{K} \alpha$ lines in our empirical fit to the ASCA spectrum are indicative of fluorescent emission. As a better model for the X-ray continuum shape we therefore use that predicted by Lightman \& White (1988) for a disk illuminated by a power law. Our data do not constrain the inclination or the solid angle subtended by the disk, so we fix these parameters at $30^{\circ}$ and $2 \pi$,

\footnotetext{
${ }^{8}$ For comparison to the constant-pressure models of Krolik \& Kriss (1995), the ionization parameter $\Xi=$ $P_{\text {rad }} / P_{\text {gas }}=5.3 U$ for gas at $10^{5} \mathrm{~K}$.
} 
respectively. The source is assumed to radiate isotropically, and we impose a high energy cutoff of $300 \mathrm{keV}$ on the intrinsic power law.

Using the optical depths given by our best empirical fit in Table 1 and the threshold photoionization cross sections of Verner \& Yakovlev (1995), we infer column densities for O VII and O VIII of $3.5 \times 10^{18} \mathrm{~cm}^{-2}$ and $2.6 \times 10^{18} \mathrm{~cm}^{-2}$, respectively. Assuming these represent all the oxygen atoms, the equivalent total hydrogen column for a solar abundance of oxygen is $9.3 \times 10^{21} \mathrm{~cm}^{-2}$. Replacing the two oxygen edges in our empirical model with the grid of warm absorber models, for the best fit we obtain a column density and ionization parameter that qualitatively matches our expectations, as shown in the center column of Table 2. This fit is only slightly worse than our empirical model. Warm absorber models computed with our alternative ionizing spectra give identical best fit values for the total column density $(\log N=22.02)$. For the hard $\nu^{-0.78}$ spectrum the best-fit ionization parameter is $U=0.25$ with $\chi^{2} / \nu=501.5 / 427$, and for the spectrum like NGC 5548, $U=1.71$ with $\chi^{2} / \nu=500.7 / 427$.

As discussed in our companion paper (Kriss et al. 1996), the strengths of the UV lines observed in NGC 3516 require a zone of lower ionization and lower column density than this warm-absorber model. Other observations also indicate that the warm absorbing medium may be complex. Otani et al. (1996) find that the O VIII opacity in MCG-6-30-15 is variable, while the O VII opacity is not, suggesting that the absorption arises in at least two different zones. Prompted by these suggestions of spectral complexity, we experimented with adding a second warm absorber to the fit. This significantly improves $\chi^{2}$. Although the level of improvement is not sufficient to be apparent in any particular feature in the spectrum, an $\mathrm{F}$ test for 3 additional parameters producing $\Delta \chi^{2}=14.2$ shows that this second model component is significant at the $99 \%$ confidence level. The parameters of this best fit are summarized in the last column of Table 2. The SIS0 and SIS1 spectra and this best fit model are illustrated in the top panel of Figure 3 .

As in Krolik \& Kriss (1995) all the warm absorber models above assumed resonant line scattering profiles with Doppler parameters given by the sound speed in the photoionized gas. Since the line opacity is sensitive to the assumed profile width, it influences the transmission computed for each model. At low velocities the opacity is dominated by continuum absorption; at high velocities resonant line scattering makes a significant contribution. To illustrate, Figure 1 shows the computed transmission for $b=200 \mathrm{~km} \mathrm{~s}^{-1}$ divided by that for $b=10 \mathrm{~km} \mathrm{~s}^{-1}$. Physical parameters as determined by the best-fit single absorber model in Table 2 were used for the ionization parameter and column density. The upper panel shows the ratio of the models themselves. The lower panel shows the ratio after the models have been folded through the ASCA response function. The error bars on each bin are taken from the corresponding data points.

The stronger predicted line absorption at $b=200 \mathrm{~km} \mathrm{~s}^{-1}$ is clear. Below $1 \mathrm{keV}$ O VIII and Fe-L transitions dominate the increased opacity. The only significant features above $1 \mathrm{keV}$ are the resonance transitions of $\mathrm{Mg}$ XI and Si XIII. At $b=10 \mathrm{~km} \mathrm{~s}^{-1}$ the absorption lines have an integrated equivalent width of $5 \mathrm{eV}$ and make a negligible contribution to the total opacity, whereas 
at $b=200 \mathrm{~km} \mathrm{~s}^{-1}$ their equivalent width is $42 \mathrm{eV}$ and $6 \%$ of the opacity between 0.6 and $2.0 \mathrm{keV}$ is due to line absorption.

Within the context of our photoionization models the dependence of line opacity on line width permits us to constrain the Doppler parameter, even though discrete absorption lines are not unambiguously present in the observed spectrum. In essence we find that the absence of significant resonant absorption line features permits us to set upper limits on the Doppler parameter. We

Table 2. Warm Absorber Model Parameters

\begin{tabular}{lcc}
\hline \multicolumn{1}{c}{ Parameter } & Single Absorber & Two Absorbers \\
& & \\
\hline$N_{H I}{ }^{\mathrm{a}}\left(10^{20} \mathrm{~cm}^{-2}\right)^{\mathrm{b}}$ & $7.1 \pm 1.7$ & $7.7 \pm 1.6$ \\
Ionization Parameter $U_{1}$ & $0.48 \pm 0.06$ & $1.66 \pm 0.31$ \\
$\log$ Total Column Density $N_{1}$ & $22.02 \pm 0.06$ & $22.15 \pm 0.12$ \\
Redshift $z_{1}$ & $0.037 \pm 0.011$ & $0.007 \pm 0.014$ \\
Ionization Parameter $U_{2}$ & $\ldots$ & $0.32 \pm 0.11$ \\
log Total Column Density $N_{2}$ & $\ldots$ & $21.84 \pm 0.04$ \\
Redshift $z_{2}$ & $\ldots .97 \pm 0.08$ & $0.050 \pm 0.013$ \\
Intrinsic Energy index $\alpha^{\mathrm{c}}$ & $0.0167 \pm 0.0014$ & $1.05 \pm 0.07$ \\
Normalization $\left(\mathrm{phot} \mathrm{cm}^{-2} \mathrm{~s}^{-1} \mathrm{keV}^{-1}\right)$ & $6.289 \pm 0.039$ & $0.0191 \pm 0.0020$ \\
Narrow Fe Energy $(\mathrm{keV})^{\mathrm{d}}$ & $76 \pm 29$ & $6.294 \pm 0.038$ \\
Narrow Fe EW $(\mathrm{eV})$ & $5.91 \pm 0.33$ & $6.00 \pm 0.34$ \\
Broad Fe Energy $(\mathrm{keV})$ & $387 \pm 170$ & $427 \pm 171$ \\
Broad Fe EW $(\mathrm{eV})$ & $2.33 \pm 0.63$ & $2.47 \pm 0.89$ \\
Broad Fe FWHM $(\mathrm{keV})$ & $500.7 / 427^{\mathrm{e}}$ & $486.5 / 424^{\mathrm{f}}$ \\
$\chi^{2} / \nu$ & & \\
\hline
\end{tabular}

a The quoted errors are $90 \%$ confidence for a single interesting parameter.

b Equivalent neutral hydrogen column for absorption by cold (neutral) gas.

c This is the intrinsic energy index for a power-law spectrum illuminating a cold disk of gas. The inclination of the disk is fixed at $30^{\circ}$ and its covering fraction is fixed at $2 \pi$.

$\mathrm{d}$ The intrinsic width of this unresolved line was held fixed at $0 \mathrm{eV}$.

e The probability of exceeding $\chi^{2}$ in this fit is 0.0079 .

f The probability of exceeding $\chi^{2}$ in this fit is 0.019 . 


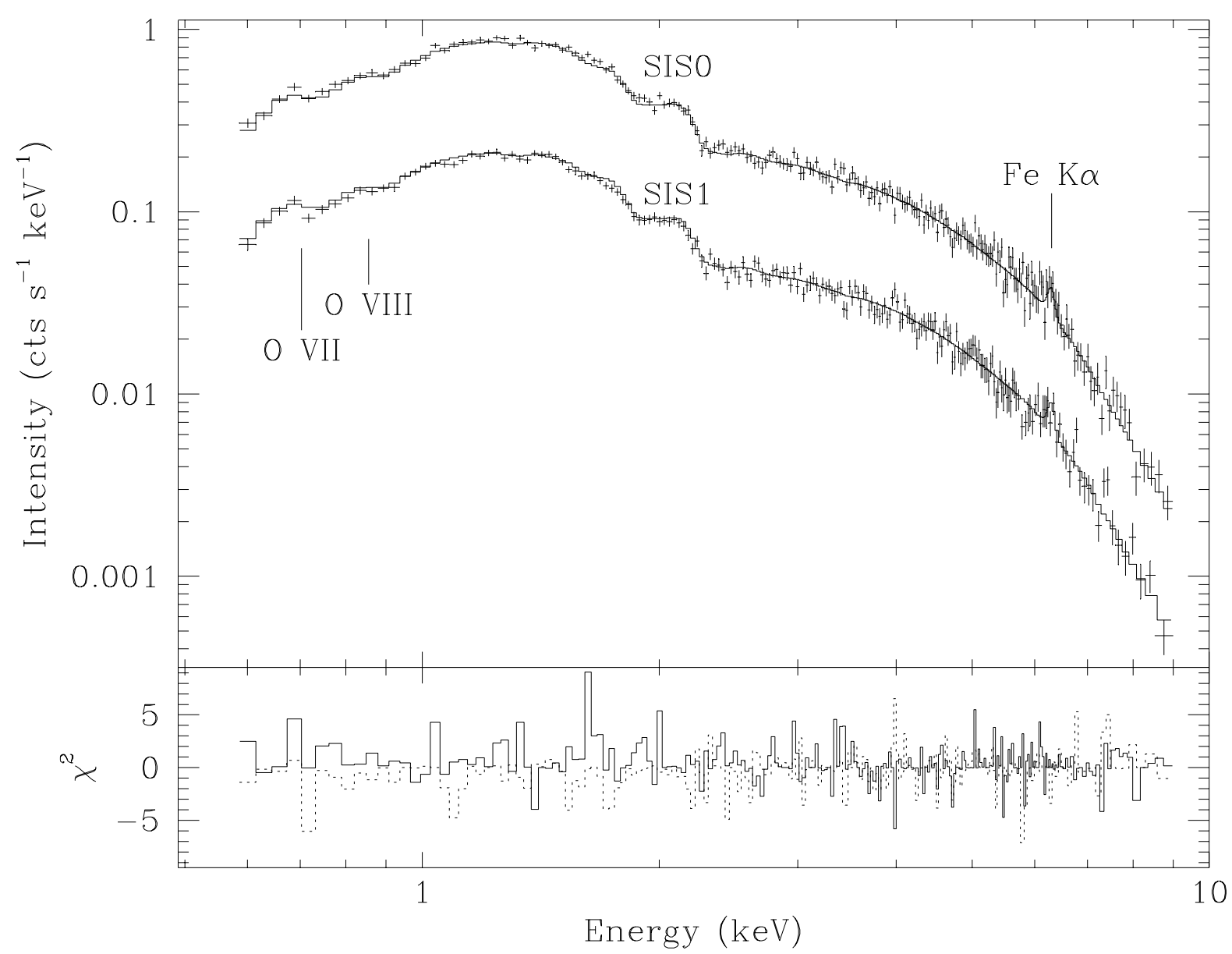

Fig. 3.- Upper Panel: The solid lines are the best-fit empirical model folded through the ASCA SIS0 and SIS1 detector responses. The data points are crosses with $1 \sigma$ error bars. The SIS1 data are offset down by 0.5 in the log for clarity. The model includes a power law with photon index 1.78 , absorption by neutral gas with an equivalent neutral hydrogen column of $\mathrm{N}_{\mathrm{H}}=6.8 \times 10^{20} \mathrm{~cm}^{-2}$, a photoionization edge due to $\mathrm{O}$ VII at $0.71 \mathrm{keV}$ with an optical depth at the edge of 0.65 , a photoionization edge due to $\mathrm{O}$ VIII at $0.86 \mathrm{keV}$ with optical depth 0.31 , an unresolved iron $\mathrm{K} \alpha$ line at $6.29 \mathrm{keV}$ with an equivalent width of $73 \mathrm{eV}$, and a broad $(\mathrm{FWHM}=1.58 \mathrm{keV})$ iron $\mathrm{K} \alpha$ line at $5.88 \mathrm{keV}$ with an equivalent width of $180 \mathrm{eV}$. Lower Panel: The contributions to $\chi^{2}$ of each spectral bin are shown. The solid line is for SIS0 and the dotted line for SIS1.

computed grids of models with Doppler parameters varying from $10 \mathrm{~km} \mathrm{~s}^{-1}$ to $200 \mathrm{~km} \mathrm{~s}^{-1}$. The best fits for both the single and the double warm absorber have Doppler parameters of $50 \mathrm{~km} \mathrm{~s}^{-1}$, approximately the sound speed for these models. (For the double warm absorber model we used the same Doppler parameter for each component.) At $90 \%$ confidence for a single interesting parameter $\left(\Delta \chi^{2}=2.706\right)$ we constrain the Doppler parameter to values less than $160 \mathrm{~km} \mathrm{~s}^{-1}$ for the single warm absorber model, and to less than $120 \mathrm{~km} \mathrm{~s}^{-1}$ for the model with two warm absorbers. 


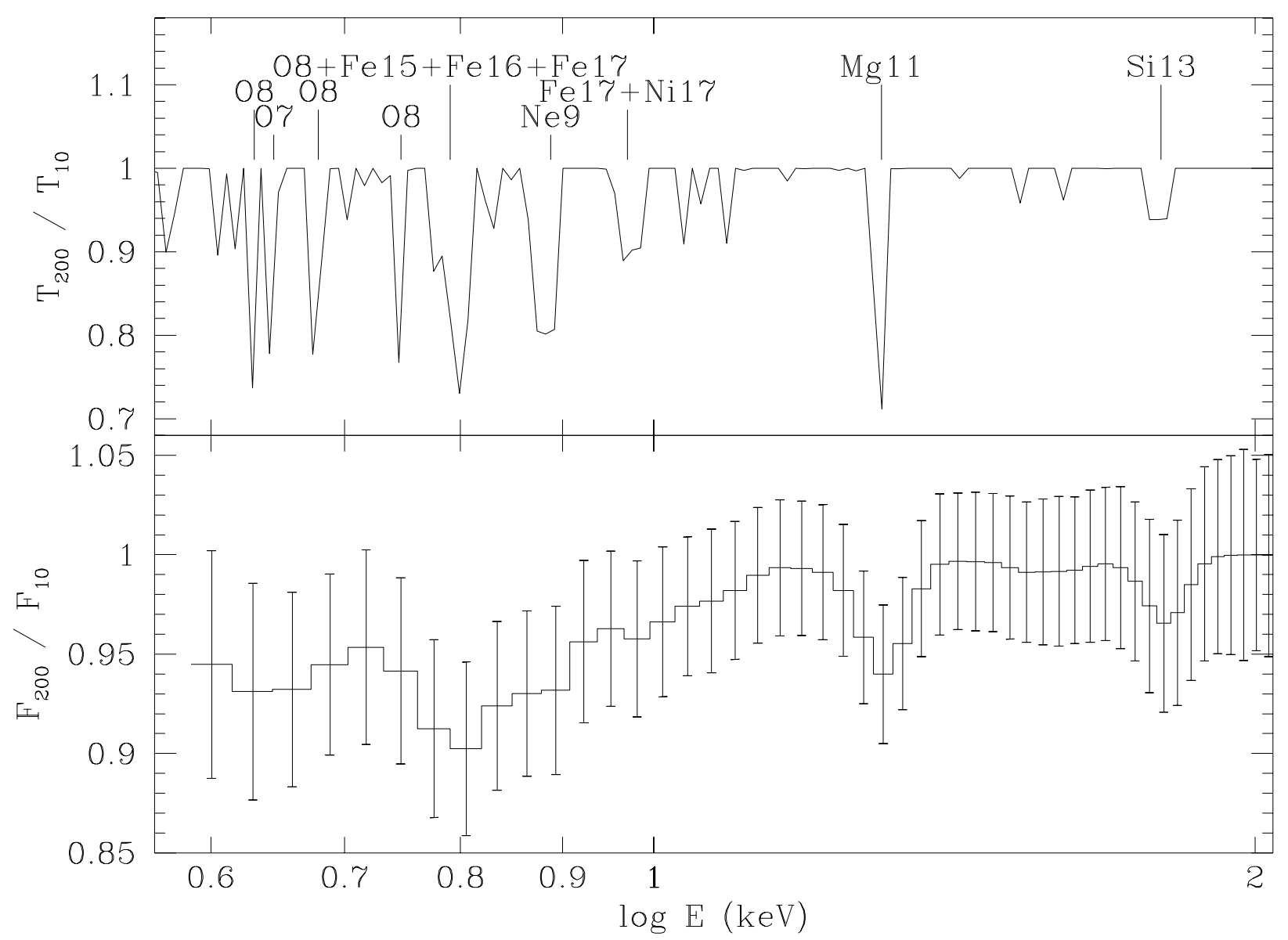

Fig. 4.- Top Panel: The ratio of the transmission for a warm absorber model with absorption lines broadened by a Doppler parameter of $b=200 \mathrm{~km} \mathrm{~s}^{-1}$ to the same model broadened with $b=10 \mathrm{~km} \mathrm{~s}^{-1}$. Physical parameters as determined by the best-fit single absorber model in Table 2 were used for the ionization parameter and column density. Ion species contributing to the most significant lines are marked. Lower Panel: Here the models are folded through the ASCA response function before computing the ratio. The error bars on each bin are taken from the corresponding data at that point.

\section{Discussion}

The broad Fe K $\alpha$ emission line in our spectrum of NGC 3516 resembles features seen in MCG6-30-15 (Tanaka et al. 1995; Fabian et al. 1995), in NGC 5548 and IC 4329A (Mushotzky et al. 1995 and in NGC 4151 (Yaqoob et al. 1995). At the signal-to-noise ratio of our spectrum we are unable to place significant constraints on relativistic disk models. In fact, the width and luminosity of the line depends strongly on our model for the underlying continuum. In the empirical model, summarized in Table 1 , the line width is smaller largely because the underlying continuum is flatter 
and has less curvature than in the warm absorber models. In the warm absorber models the widths and equivalent widths are large, but given the uncertainties, they are compatible with the maximum equivalent widths of $\sim 200 \mathrm{eV}$ expected in models of X-ray reflection from cold disks (e.g., George \& Fabian 1991.

A simple, single-zone photoionized absorber is an imperfect description of the soft X-ray opacity in NGC 3516. At least two zones are required to give as good a fit to the data as our empirical model containing discrete absorption edges due to $\mathrm{O}$ VII and $\mathrm{O}$ vIII. These two zones may be a simplification of a broad distribution of ionization parameters in the absorbing gas, or they may be indicative of two entirely different regions as suggested by Otani et al. (1996) in their study of the variability of $\mathrm{O}$ VII and $\mathrm{O}$ VIII opacity in MCG-6-30-15. The complexity of the absorbing gas in NGC 3516 increases even more when one considers the UV absorption lines observed simultaneously with HUT. In the companion to this paper Kriss et al. (1996) find that neither a single photoionized absorber nor the multiple warm absorber models considered here are adequate to explain the UV absorption lines. This confirms the conclusion of Kolman et al. (1993) that there is probably not a direct connection between the warm X-ray and the UV absorbers in NGC 3516.

Having separate regions for the X-ray and UV absorption contrasts with the conclusions of Mathur et al. $(1994,1995)$ in their studies of the warm absorbers in 3C 351 and in NGC 5548. For those objects they described a single absorbing zone that could account for both the X-ray warm absorber and the associated UV absorption lines. Not all X-ray and UV absorbers may be so simple. A clue to the additional complexity of the absorption in NGC 3516 is the presence of large columns of lower ionization species such as Si IV and an optically thick Lyman limit. Low ionization species also present problems for single zone models in attempting to model UV and X-ray absorption in NGC 4151 (Kriss et al. 1995). Since Si IV absorption is strong in broad absorption line quasars, by analogy this may indicate that single zone models will also not suffice to explain both the UV and X-ray absorption in these objects, contrary to the suggestion of Mathur, Elvis, \& Singh (1995).

Although the absorbing medium in NGC 3516 appears to be highly stratified, the presently observed weak X-ray absorption coincident with an episode of weak UV absorption suggests that some underlying mechanism ties them together. At the epoch of our observation, NGC 3516 was brighter than usual by about a factor of two, but the low energy X-ray absorption decreased by more than can be accounted for simply by photoionization due to the increased luminosity. As suggested by Walter et al. (1990), these large changes in absorption column may be caused by different clouds moving across the line of sight. Rather than a single cloud, however, we require a whole population of clouds of differing column densities and ionization parameters moving into place. If the absorption arises in a wind driven from the accretion disk or the obscuring torus, large changes in opacity that are correlated in the UV and the X-ray may be linked by fluctuations in the mass supply to the outflow. X-ray and UV absorption in AGN may ultimately have a common origin, but the absorption probably occurs in distinctly different regions with a variety of physical conditions. 
This work was supported by a NASA ASCA guest investigator grant NAG 5-2935 and a NASA

Long-Term Space Astrophysics grant NAGW-4443 to the Johns Hopkins University. CO thanks the Special Postdoctoral Researchers Program of RIKEN for support.

\section{REFERENCES}

Burke, B. E., et al., 1994, IEEE Trans. Nuc. Sci., 41, 375

Fabian, A. C., et al. 1994, PASJ, 46, L59

Fabian, A. C., et al. 1995, MNRAS, in press

George, I., \& Fabian, A. C. 1991, MNRAS, 249, 352

Halpern, J. P., 1982, Ph.D. thesis, Harvard University

Halpern, J. P., 1984, ApJ, 281, 90

Holt, S. S., et al. 1980, ApJ, 241, L13

Lightman, A. P., \& White, T. R. 1988, ApJ, 335, 57

Kolman, M., Halpern, J. P., Martin, C., Awaki, H., \& Koyama, K. 1993, ApJ, 403, 592

Koratkar, A., et al. 1996, in preparation

Kriss, G. A., \& Canizares, C. R. 1985, ApJ, 297, 177

Kriss, G. A., Davidsen, A. F., Zheng, W., Kruk, J. W., \& Espey, B. R. 1995, ApJ, 454, L7

Kriss, G. A., et al. 1996, ApJ, this issue

Krolik, J. H., \& Kriss, G. A. 1995, ApJ, 447, 512

Makishima, K. et al. 1996, PASJ, submitted

Mathur, S., Elvis, M., \& Singh, K. P. 1995, ApJ, 455, L9

Mathur, S., Wilkes, B., Elvis, M., \& Fiore, F. 1994, ApJ, 434, 493

Mathur, S., Wilkes, B., \& Elvis, M. 1995, ApJ, 452, 230

Mushotzky, R. F., Fabian, A. C., Iwasawa, K., Kunieda, H., Matsuoka, M., Nandra, K., \& Tanaka, Y. 1995, MNRAS, 272, L9

Nandra, P., \& Pounds, K. A. 1994, MNRAS, 268, 405

Netzer, H. 1993, ApJ, 411, 594 
Otani, C., et al. 1996, PASJ, in press

Otani, C., et al. 1996, in Röntgensstrahlung from the Universe, eds. Zimmermann, H.U., Trümper, J., \& Yorke H. (Garching: MPE) in press

Reynolds, C. S., \& Fabian, A. C. 1995, MNRAS, 273, 1167

Serlemitsos, P. J., et al., 1995, PASJ, 47, 105

Shafer, R. A., Haberl, F., \& Arnaud, K. A. 1989, XSPEC: An X-ray Spectral Fitting Package (ESA TM-09) (Paris: ESA)

Stark, A.A., et al. 1992, ApJS, 79, 77

Tanaka, Y., et al. 1995, Nature, 375, 659

Tanaka, Y., Holt, S.S., \& Inoue, H. 1994, PASJ, 46, L37

Tarter, C. B., Tucker, W. H., \& Salpeter, E. E. 1969, ApJ, 156, 943

Turner, T.J., Nandra, K., George, I.M., Fabian, A.C., \& Pounds, K.A. 1993, ApJ, 419, 127

Ulrich, M.-H., \& Boisson, C. 1983, ApJ, 267, 515

Verner, D. A., \& Yakovlev, D. G. 1995, A\&AS, 109, 125

Voit, G. M., Shull, J. M., \& Begelman, M. C. 1985, ApJ, 316, 573

Vrtilek, J. M., \& Carleton, N. A. 1985, ApJ, 294, 106

Walter, R., Ulrich, M.-H., Courvoisier, T. J.-L., \& Buson, L. M. 1990, A\&A, 233, 53

Yaqoob, T., Warwick, R. S., \& Pounds, K. A. 1989, MNRAS, 236, 153

Yaqoob, T., et al. 1993, MNRAS, 262, 435

Yaqoob, T., Edelson, R., Weaver, K. A., Warwick, R. S., Mushotzky, R. F., Serlemitsos, P. J., \& Holt, S. S. 1995, ApJ, 499, L99 